\title{
Correction to: Amycolatopsis pittospori sp. nov., an endophytic actinobacterium isolated from native apricot tree and genome mining revealed the biosynthesis potential as antibiotic producer and plant growth promoter
}

\author{
Onuma Kaewkla Christopher Milton Mathew Franco
}

Published online: 8 December 2021

(C) Springer Nature Switzerland AG 2021

Correction to: Antonie van Leeuwenhoek (2021) 114:365-377 https://doi.org/10.1007/s10482-021-01519-5

In the original publication of the article, the culture collection numbers were incorrectly published as "DSM 101857T = NRRL B-65350T" in the protologue on page 375 .
The correct collection numbers are "= NRRL B-65536T = TBRC 10618T”.

Publisher's Note Springer Nature remains neutral with regard to jurisdictional claims in published maps and institutional affiliations.

The original article can be found online at https:// doi.org/10.1007/s10482-021-01519-5.

O. Kaewkla $(\square)$

Department of Biology, Faculty of Science, Mahasarakham University, Kantaravichai, Maha Sarakham Province 44150, Thailand e-mail: Onuma.K@msu.ac.th

O. Kaewkla · C. M. M. Franco Medical Biotechnology, College of Medicine and Public Health, Flinders University, Bedford Park,

SA 5042, Australia 